Gefässchirurgie $2007 \cdot 12: 238-242$

DOI 10.1007/s00772-007-0532-1

Online publiziert: 28. Juni 2007

(c) Springer Medizin Verlag 2007
M.K. Widmer ${ }^{1} \cdot$ I. Baumgartner ${ }^{2} \cdot$ T. Carrel $^{1} \cdot$ J. Schmidli ${ }^{1}$

${ }^{1}$ Klinik und Poliklinik für Herz- und Gefässchirurgie, Inselspital, Bern

${ }^{2}$ Klinik und Poliklinik für Angiologie, Inselspital, Bern

\title{
Die endovaskuläre Ausbildung für Gefäßchirurgen an der Universitätsklinik Bern
}

Die optimale Ausbildung zum künftigen Gefäßchirurgen lässt sich auf dem Papier problemlos theoretisch festhalten. In der Praxis aber sieht es häufig ganz anders aus. Die Ausbildung ist stark von der historischen Entwicklung im Krankenhaus und den örtlichen Gegebenheiten abhängig. Abläufe und Verfahren, die uns heute optimal erscheinen, müssen vielleicht morgen schon hinterfragt und angepasst werden. Die Prozesse sind untrennbar verknüpft mit dem Faktor Mensch, denn dieser muss seine Haltung ändern, sich anpassen, von Positionen abrücken, neue Evidenz akzeptieren und neue Fähigkeiten durch sorgfältiges Training erarbeiten. Dies ist nur möglich, wenn in der interdisziplinären und interprofessionellen Arbeitsgemeinschaft gegenseitige Achtung und Respekt vorherrschen und jedem das gemeinsame Ziel bekannt ist, nämlich die optimale Betreuung von Patienten mit kardiovaskulären Erkrankungen sicherzustellen.

\section{Die Einführung der endovaskulären Verfahren in Bern}

Mit der ersten Ballondilatation von Koronararterien durch den Angiologen und Kardiologen Andreas Grüntzig in Zürich hat die endovaskuläre Therapie seit 1977 in der ganzen Welt einen ungeahnten Aufschwung genommen. Felix Mahler, einer der Pioniere auf dem Gebiet der peripheren endovaskulären Therapie, hat die
Expertise von Zürich nach Bern gebracht und zusammen mit dem Institut für Radiologie etabliert [5]. Vor einer Katheterintervention oder einer Gefäßoperation brauchen Patienten jedoch v. a. eine kompetente diagnostische Abklärung, wozu der Duplexultraschall zur Planung unentbehrlich ist. Außerdem braucht es internistische Fachkenntnisse, um eine optimale Therapie der kardiovaskulären Risikofaktoren zu garantieren. Die oben genannten Aufgaben lassen sich durch eine gut funktionierende angiologisch-gefäßchirurgische Poliklinik bewältigen, wie sie Bern seit Jahren kennt.

\section{Die Departementalisierung am Inselspital 1999}

Im Jahre 1999 wurde das „organspezifische“ Departement „Herz und Gefäße “ am Inselspital Bern geschaffen. Dazu gehören die Kliniken für Kardiologie, Herzund Gefäßchirurgie sowie Angiologie. Damit war es möglich, organisatorische und administrative Verbesserungen zu realisieren, die dank kurzer Wege eine interdisziplinäre Therapie täglich rund um die Uhr an 365 Tagen im Jahr erlauben. Gemeinsame fachliche und wissenschaftliche Interessen von Ärzten und Ärztinnen mit unterschiedlichen Ausbildungsprofilen, ein gemeinsamer Budgetprozess und gemeinsame Pflegestandards haben wesentlich zum Erfolg des neu geschaffenen Kompetenzzentrums beigetragen. Dem Universitätsspital Bern mit seinen logistischen und apparativen Kapazitäten kommt große Bedeutung zu, da es
Notfallpatienten oder solche mit komplexen Gefäßpathologien von den umliegenden Spitälern und den frei praktizierenden Gefäßspezialisten übernimmt. Die Klinik und Poliklinik für Herz- und Gefäßchirurgie hat mit ihrer Infrastruktur von 56 Betten (davon 8 für „Intermediate Care") die Versorgung von rund 1,5 Mio. Menschen mit komplexen Gefäßerkrankungen sicherzustellen.

Im Jahre 2006 haben 5 Kaderärzte (Abteilungsleiter und Stellvertreter, 3 Oberärzte) und 2 Fachassisten(inn)en mit dem Ausbildungsziel Gefäßchirurgie EBSQVASC rund 1200 Eingriffe durchgeführt. Wöchentliche Fortbildungsveranstaltungen auf Departementsstufe, klinikinterne Weiterbildungen, tägliche Rapporte mit den Angiologen und Radiologen und interdisziplinäre Diskussionen auf der angiologisch-gefäßchirurgischen Poliklinik sind wichtig, um lege artis die Interventions- bzw. die Operationsindikationen unter Abschätzung der Risiken des einen oder anderen Verfahrens festzulegen.

\section{Ausbildungsvorgaben der FMH (rechtliche Absicherung, Europanormen)}

Gefäßchirurgische Anwärter mit dem "Schweizerischen Facharzt für Chirurgie“ haben zur Erlangung des Schwerpunkttitels „Gefäßchirurgie“ der Foederatio Medicorum Helveticorum (FMH) die Vorgaben des USMM zum „Europäischen Exa-

In diesem Artikel wird die männliche Form verwendet, welche aber für beide Geschlechter gilt. 
Hier steht eine Anzeige.

第 Springer 


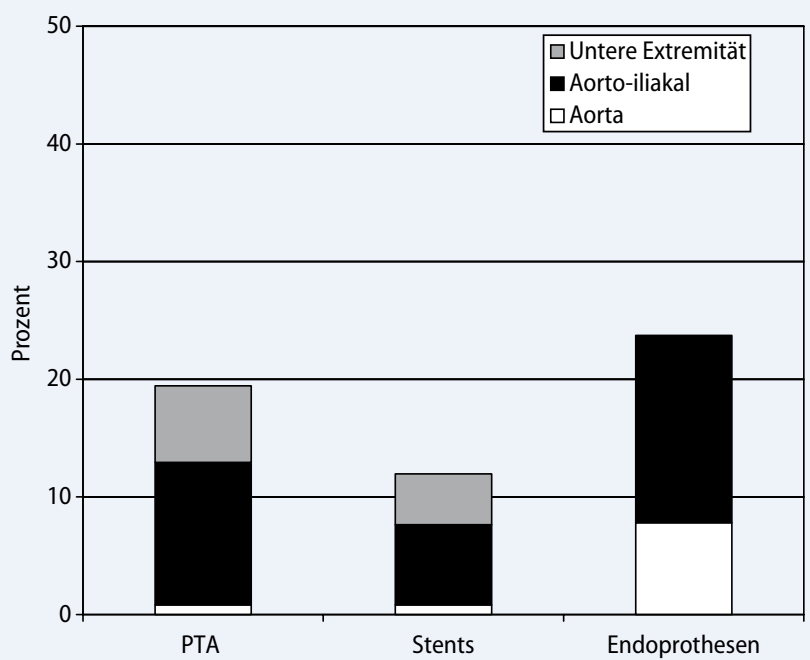

Abb. $1 \Delta$ Anteil endovaskulärer Verfahren bei offenen Eingriffen im Bereich Gefäße der unteren Extremitäten, Aorta und aortoiliakale Gefäßachse im Jahre 2006 an der Klinik und Poliklinik für Herz- und Gefäßchirurgie am Inselspital Bern

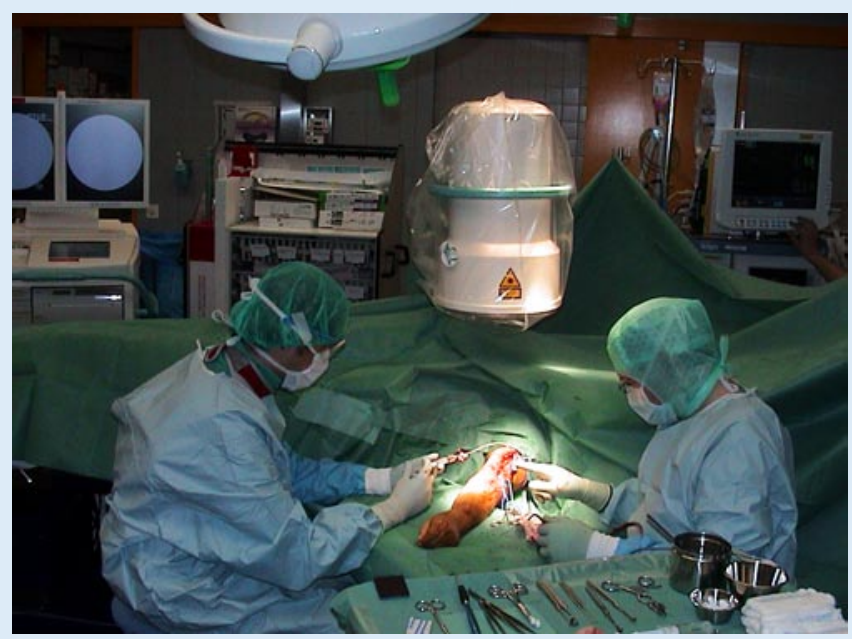

Abb. $2 \Delta$ Einrichtung des Operationssaales mit Röntgenanlage und Materialwagen, die standardisierte Sets für kathetertechnische Interventionen enthalten. Im Bild: Revision einer verschlossenen Oberarmfistel (V.-cephalica-Fistel) mit zentraler Stenose der V. subclavia
Tab. 1 Übersicht über intraoperativ durchgeführte kathetertechnische Interventionen

- Endograftimplantationen bei Aortenpathologien

- Hybridverfahren im Aortenbogenbereich

- Intraoperative Kombinationsverfahren: offene femorale Thrombendarterektomie mit Stenting der Beckenachse bzw. PTA der Peripherie

- Venöse Thrombektomie mit Stenting einer Beckenvenenstenose

- Femorale Embolektomie mit Katheteraspiration, evtl. kombiniert mit lokaler Lyse

- Additive Katheterinterventionen bei Fistelrevisionen von Hämodialysepatienten

- Angioskopie und angioskopisch assistierte Valvulotomie beim autologen Venenbypass

men EBSQ-VASC“ zu erfüllen [6]. In diesem Anforderungsprofil werden endovaskuläre Fertigkeiten wie PTA und Implantationen von Stents oder Stentgrafts verlangt. Im Herbst 2006 hat die Schweizerische Gesellschaft für Chirurgie im Curriculum für den Schwerpunkttitel „Gefäßchirurgie" eine Grundausbildung in endovaskulären Techniken vorgeschrieben, wozu die Zustimmung anderer Fachgesellschaften notwendig war.

Diese nun neu beschlossenen Vorgaben implizieren eine Änderung des Ausbildungsspektrums, wobei bei der Umsetzung eine enge Kooperation mit Nachbarkliniken notwendig ist. So wie jeder junge
Gefäßchirurg sich Kenntnisse im vaskulären Ultraschall anzueignen hat, muss er auch eine Grundausbildung in endovaskulären Techniken durchlaufen. Bei Interventionen im Operationssaal hat die Verantwortung bei den Chirurgen zu liegen. - Abb. 1 zeigt den Anteil an Gefäßeingriffen mit kathetertechnischen Interventionen, die bei uns im Jahre 2006 im Operationssaal durchgeführt wurden.

\section{Materielle Bereitschaft im Operationssaal erstellen}

Um jederzeit im Operationssaal interventionelle Eingriffe durchführen zu können, muss der Operationssaal systematisch mit einem kompletten Sortiment an endovaskulären Instrumenten, Stents und Endoprothesen aufgerüstet werden. Es wurden deshalb Sets mit Interventionsmaterial zusammengestellt, die auch weniger erfahrenen Operationsschwestern eine kompetente Mitwirkung erlauben und so lästige Wartezeiten vermindern helfen $\bullet$ Abb. 2. Mit dem nun verfügbaren Material lassen sich intraoperative Katheteraspirationen, Dilatationen oder Stentimplantationen von Beckenarterien oder nach venöser Thrombektomie durchführen. Es können aber auch rupturierte Bauchaortenaneurysmen, akute Aortendissektionen und traumatische Aortenrupturen unverzüglich im Operationssaal versorgt werden.
Die bisherigen mobilen Bildverstärkeranlagen werden in diesem Jahr durch neue, bedienerfreundliche Durchleuchtungsgeräte ersetzt. Diese nun vorhandene materielle Autonomie ist eine Grundvoraussetzung, um kathetertechnische Interventionen im Operationssaal durchzuführen. Bei den interventionellen Eingriffen werden nach Absprache immer Angiologen oder Radiologen hinzugezogen.

\section{Ausbildung von Assistenzpersonal}

Nachdem sich die medizinisch-technischen Röntgenassistenten wegen Personalengpässen von der Bedienung des Bildverstärkers und des Kontrastmittelinjektors zurückgezogen haben, wurden 2 Operationspfleger speziell dafür ausgebildet, diese Geräte zu bedienen. Diese Personen werden seit 2006 im Routineprogramm eingesetzt, wenn Endoprothesen implantiert werden. Sie stehen aber auch auf Abruf für die Versorgung von BDissektionen und rupturierten Bauchaortenaneurysmen zur Verfügung. Mit einer Intensivierung der endovaskulären Aktivität müssen die Operationsschwestern vermehrt in diesen Techniken instruiert werden. Dieser Weiterbildung des Operationspersonals schenken wir große Beachtung, damit die endovaskulären Interventionen reibungslos durchgeführt werden können. 


\section{Endovaskuläre Fertigkeiten im Operationssaal}

In der heutigen Versorgung von Patienten mit Gefäßerkrankungen gibt es eine Unzahl von Eingriffen, die wir im Operationssaal mit einem kathetertechnischen Armamentarium durchführen. In - Tab. 1 sind diese Eingriffe aufgelistet.

Die intraoperativen Angiographien zur Kontrolle von Bypassneuanlagen oder zur Beurteilung der Qualität nach Revisionen von peripheren Bypässen bzw. nach Revisionseingriffen von Fisteln bei Hämodialysepatienten sind unverzichtbare Eingriffe, um Sicherheit in der Handhabung der Röntgenanlage zu bekommen. Die "Over-the-wire“-Embolektomie mittels Fogarty-Katheter unter Durchleuchtung erlaubt eine schonendere Manipulation in den Unterschenkelgefäßen. Außerdem kann die senkrecht abgehende A. tibialis anterior kanalisiert werden, und der Therapieerfolg wird nicht nur klinisch durch Beurteilung des Backflows, sondern auch angiographisch dokumentiert.

Bei Patienten, die eine obstruierende Plaque im Femoralbereich aufweisen, lassen sich nach einer Endarterektomie, in der gleichen Sitzung auch Inflow-Probleme mit Stents versorgen oder periphere Stenosen dilatieren.

Bisher haben wir aortale Endografts immer im Operationssaal in Zusammenarbeit mit Radiologen und Angiologen implantiert. Die erfolgversprechenden Resultate bei rupturierten Bauchaortenaneurysmen und der Trend, Komplikationen von B-Dissektionen mit Stentgrafts zu versorgen, machen es nötig, dass die entsprechenden Implantate im Operationssaal zur Verfügung stehen [3]. Diese logistische Leistung ist zwingend, um ohne jede Verzögerung operieren zu können.
Hybridverfahren im Bereich des Aortenbogens, venöse Thrombektomien mit Stenting eines Beckensporns und die Versorgung von Gefäßläsionen bei Traumapatienten sind weitere Eingriffe, bei denen der Chirurg seine kathetertechnischen Fertigkeiten anwenden kann $[1,4]$.

\section{Wohin wollen wir gehen?}

Heute lässt sich mit wenig Aufwand und guten Resultaten eine ganze Reihe von kathetertechnischen Eingriffen als additive Verfahren im Operationssaal durchführen. Damit kann die Qualität und möglicherweise auch die Prognose einer chirurgischen Rekonstruktion nachhaltig verbessert werden. Es lassen sich Patienten mit komplexen oder multifokalen Gefäßläsionen in einer Sitzung behandeln.

Eine gute Kollaboration mit interventionell tätigen Angiologen und Radiolo-

\section{Hier steht eine Anzeige.}

\section{Springer}


Gefässchirurgie $2007 \cdot$ 12:238-242

DOI 10.1007/s00772-007-0532-1

(c) Springer Medizin Verlag 2007

M.K. Widmer · I. Baumgartner · T. Carrel · J. Schmidli

Die endovaskuläre Ausbildung

für Gefäßchirurgen an der

Universitätsklinik Bern

Zusammenfassung

Die technische Entwicklung im Bereich von Katheterinterventionen hat auch zunehmenden Einfluss auf das Tätigkeitsfeld der Gefäßchirurgen. Im Operationssaal werden häufiger additive Katheterinterventionen oder Kombinationsverfahren, auch $\mathrm{Hy}$ brideingriffe genannt, durchgeführt. Dies setzt zwingend kathetertechnische Fertigkeiten voraus. Deshalb werden an der Klinik und Poliklinik für Herz- und Gefäßchirurgie in Bern in Zusammenarbeit mit interventionell tätigen Angiologen und Radiologen die Gefäßchirurgen in kathetertechnischen Interventionen trainiert. Katheterinterventionen zwingen dazu, die technische Ausrüstung des Operationssaals laufend anzupassen und die Schulung des Assistenzpersonals zu fördern.

Schlüsselwörter

Gefäßchirurgie · Endovaskuläre Verfahren .

Ausbildung

Training in endovascular techniques for vascular surgeons at the University Hospital in Bern

\begin{abstract}
The technical developments in the area of catheter-based interventions are influencing vascular surgeons' daily practice more and more. In the operating theatre combined open and endovascular interventions, socalled hybrid procedures, are frequently performed, and meticulous endovascular skills are essential for these. In the Clinic for Cardiovascular Surgery in Bern the training for vascular surgeons involves the cooperation of interventionalists with backgrounds in either angiology or radiology. In addition, the technical equipment in the operating room must be continuously updated and the ancillary staff need more practical training.
\end{abstract}

Keywords

Vascular surgery - Endovascular treatment . Training gen ist deshalb wünschbar, doch muss im Operationssaal die Führung beim Chirurgen liegen. Dies ist notwendig, damit sich der Gefäßchirurg auch in Zukunft zum Gefäßspezialisten weiterentwickeln kann, wie es in England durch eine gemeinsame Absichtserklärung zur engeren Kooperation zwischen Interventionalisten und Gefäßchirurgen modellhaft bereits der Fall ist (J. Wolfe, pers. Mitteilung).

Auch die Gefäßchirurgie ist einem stetigen Wandel unterworfen. Der Trend der letzten Jahre zu mehr Katheterinterventionen wird sich fortsetzen. Will die Gefäßchirurgie mit der Entwicklung Schritt halten, muss die kommende Generation von Gefäßchirurgen ohne Ausnahme eine Grundausbildung in endovaskulären Techniken (z. B. durch Rotationen bei interventionell tätigen Gefäßchirurgen, Angiologen oder Radiologen) erhalten und vermehrt Trainingseinheiten am Simulator absolvieren. Strahlenschutzkurse und Kenntnisse über allgemeinen Strahlenschutz im Operationssaal sind für ihn ebenso zwingend wie für alle anderen interventionellen Fachgebiete.

Die oben gemachten Ausführungen gelten auch für den Herzchirurgen: Wollen sie künftig die endovaskuläre Versorgung von Aortenbogen- oder Aorta-descendens-Rupturen durchführen, müssen auch sie mittelfristig endovaskuläre Techniken oder gar kardiologische Interventionen erlernen. Sonst wird die perkutane endovaskuläre Herzklappenimplantation ohne Beteiligung des Chirurgen erfolgen.

\section{Fazit für die Praxis}

Durch Kooperation und interdisziplinäre Zusammenarbeit lassen sich die endovaskulären Ausbildungsziele für Gefäßchirurgen am besten umsetzen. Ausgehend von gemeinsamen Grundkenntnissen kann jeder an endovaskulären Interventionen beteiligte Spezialist sein spezifisches Fachwissen einbringen, um eine optimale, evidenzbasierte und ökonomische Patientenbetreuung sicherzustellen. Daran wollen wir in Bern in Zukunft weiter arbeiten.

\section{Korrespondenzadresse}

Dr. M.K. Widmer

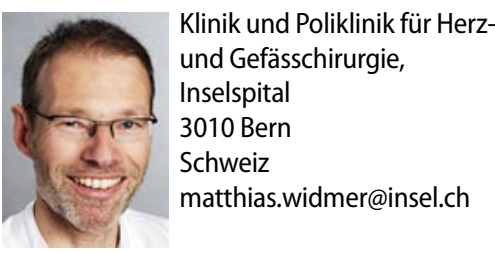

Danksagung. Wir bedanken uns bei Frau B. Gahl für die Datenanalyse.

Interessenkonflikt. Es besteht kein Interessenkonflikt. Der korrespondierende Autor versichert, dass keine Verbindungen mit einer Firma, deren Produkt in dem Artikel genannt ist, oder einer Firma, die ein Konkurrenzprodukt vertreibt, bestehen. Die Präsentation des Themas ist unabhängig und die Darstellung der Inhalte produktneutral.

\section{Literatur}

1. Blättler W, Heller G, Largiader J et al. (2004) Combined regional thrombolysis and surgical thrombectomy for treatment of iliofemoral vein thrombosis. JVasc Surg 40: 620-625

2. European Board of Surgery: http://www.uemssurg.org/vascsurg.html

3. Hechelhammer L, Lachat ML, Wildermuth $S$ et al. (2005) Midterm outcome of endovascular repair of ruptured abdominal aneurysms. J Vasc Surg 41: 752-757

4. Lutz HJ, Do DD, Schroth G et al. (2002). Hybrid therapy of symptomatic stenosis of the innominate artery. Eur J Vasc Endovasc Surg 24: 184-185

5. Mahler F, Gluck Z, Probst P et al. (1982) Percutaneous transluminal dilatation of renal artery stenosis: methods and results. Vasa 11: 353-357

6. Schweizerische Gesellschaft für Gefässchirurgie: http://www.swissvasc.ch 